\title{
Time optimization for radius updates in zone dynamics of zone routing protocol
}

\author{
Muhsin Bayu Aji Fadhillah*1, Radityo Anggoro² \\ Informatics Department, Institut Teknologi Sepuluh Nopember, Indonesia ${ }^{1,2}$
}

\author{
Article Info \\ Keywords: \\ Vehicular Ad Hoc Networks (VANETs), Zone \\ Routing Protocol (ZRP), Zone Dynamics, \\ Radius Update Time, Radius Update Time \\ Optimization
}

\section{Article history:}

Received 11 April 2020

Revised 28 April 2020

Accepted 07 May 2020

Available 15 May 2020

Cite:

Fadhillah, M., \& Anggoro, R. (2020). Time Optimization for Radius Updates in Zone Dynamics of Zone Routing Protocol. Kinetik:

Game Technology, Information System,

Computer Network, Computing, Electronics, and Control, 5(2)

doi:https://doi.org/10.22219/kinetik.v5i2.1064

${ }^{*}$ Corresponding author.

Muhsin Bayu Aji Fadhillah

E-mail address:

muhsin21baf@gmail.com

\begin{abstract}
Vehicular ad hoc networks are wireless network technologies that can be used to communicate without the need for fixed infrastructure. The use of zone routing protocol which is a hybrid routing protocol in a vehicular ad hoc network environment can reduce delay, packet flooding, and excess bandwidth usage on the network. However, traditional zone routing protocol is only configured for one fixed radius value, which makes it not adapt to existing network conditions. Zone dynamics with adaptive radius values in zone routing protocol are used so that zones formed by nodes are more optimal. In adapting the radius value to make the zone dynamics necessary, the optimal configuration of the radius update time is required. Simulations and tests that have been carried out with NS-2 show that the proper update time can improve zone routing protocol performance with a low end-to-end delay and routing overhead value, but has a high packet delivery ratio.
\end{abstract}

\section{Introduction}

Mobile Ad Hoc Networks (MANETs) are wireless networks without a fixed infrastructure that make each device can be configured independently to communicate with each other wirelessly [1][2]. MANETs are the choice for communication and information exchange in environments without fixed infrastructure such as war or conflict zones, military zones, disaster-prone areas, to areas that are difficult to reach by communication infrastructure [3]. Vehicular Ad Hoc Networks (VANETs) are a development of MANETs but are applied in a very dynamic transportation environment, where VANETs technology integrates the use of WLANs and cellular technology combined with communication between smart vehicles so that they can be used to improve the safety and efficiency of vehicle traffic [4]. VANETs are as well have several constraints such as driver habits, mobility limitations and high mobility speeds [5].

Routing protocols on ad hoc networks can be classified into three categories, namely proactive, reactive, and hybrid routing protocols [6]. Zone Routing Protocol (ZRP) itself is one type of hybrid routing protocol that combined the advantages of proactive and reactive protocols by maintaining a zone topology map centered on each node [7]. The main feature of the ZRP protocol is its dynamic behavior which adjusts the behavior of the schema from a purely reactive protocol to a proactive protocol with a radius value parameter that determines the size of the zone formed by each node [8]. In general, ZRP works proactively using IntraZone Routing Protocol (IARP), while InterZone Routing Protocol (IERP) is assisted with Bordercast Routing Protocol (BRP) to be used when ZRP works reactively [9]. In ZRP, the use of BRP in IERP can avoid excessive requests in the zone routing process, thereby reducing the number of packets sent and not burdening traffic on the network [10].

Pearlmann and Haas stated that ZRP can be configured by adjusting the radius parameters for certain networks [11]. Yélémou et al. has conducted a series of tests to find the optimal radius value by measuring ZRP performance [12], the test still used the traditional ZRP but from the result it can be concluded that the proper radius value can help improve the performance of ZRP. Chenn-Jung Huang et al. use evolving fuzzy neural networks to make the radius of the route zone in each routing table dynamically adjusted [13]. Samar, Pearlman and Haas developed Independent Zone Routing (IZR) with adaptive and distributed configurations for the optimal size of the routing zone for each node [14]. Yang et al. make a zone clustering on ZRP by selecting Cluster Head (CH) [15]. Oigawa and Sato making improvements in IntErzone Routing Protocol of ZRP based on bloom Filters [16].

In the ZRP protocol, the radius value becomes an important factor because the size of the zone formed is determined by the radius value. Traditional ZRP use a fixed radius value so it is less adaptable to existing network

Cite: Fadhillah, M., \& Anggoro, R. (2020). Time Optimization for Radius Updates in Zone Dynamics of Zone Routing Protocol. Kinetik: Game Technology, Information System, Computer Network, Computing, Electronics, and Control, 5(2). doi:https://doi.org/10.22219/kinetik.v5i2.1064 
conditions. The radius value is a single factor as a reference for the formation of zones by each node so that if a zone is formed with a radius value that is too large then too much routing table information must be updated. Conversely, if the radius value is too small, the zone may not be able to accommodate enough nodes so that there can be increased delay and packet flooding during the route search process. Various studies that have been conducted have shown that ZRP can be developed so that it can adapt to network conditions by finding optimal radius or zoning values. In the case of zone dynamics or radius value updates, the appropriate update time settings are required. Update time that is too long will make sensitivity decrease because of a very dynamic environment and too fast update time can also result in the amount of information that is too much sent so that it can flood the traffic.

This paper proposes optimization radius update time in the process of zoning dynamics in ZRP. Optimizing the time for radius update needs to be done in order to further optimize the performance of ZRP that is able to adapt to network conditions by forming zone dynamics. Zone dynamization is done by creating a radius value that is adaptive to existing network conditions. The factors that can be used as a reference to network conditions are node speed [17][18], node density [19][20][21][22], and movement direction [23]. Configuring the time to update the radius value is done in an effort to adapt the radius value to the network conditions so that ZRP performance does not produce high routing overhead or end-to-end delay but has a high packet delivery ratio. This can happen if the radius update time is neither too fast nor too slow.

\section{Research Method}

This paper proposes the optimization radius update time in zone dynamics of ZRP. Modified from traditional ZRP, zone dynamics are carried out by changing or updating the radius value based on existing network conditions. Predicting Node Trend (PNT) is a method used by Shen et al. to find the best route by considering aspects of behavior or characteristics of each node [24]. The formula from the study of Shen et al. used in this paper to calculate a threshold value based on network conditions by numbers based on factors.

Factors used as a representation of network conditions such as the quality of links from each node, speed and acceleration of the node [17][18], and node movement directions [23]. In addition to these factors, the density of neighboring nodes can also be used as an additional factor to assess the condition of the existing network [19][20][21][22]. Therefore, in this paper the density level of neighboring nodes is added as a representation of network conditions to be a reference in dynamizing zones by updating the radius value within a certain time period. The formula used also refers to the research of Arifiyan, Djanali and Anggoro which adds a factor of the density of neighboring node levels in the formula proposed by Shen et al. [25] as shown in Equation 1.

$$
T W R=f_{s} \times\left|S_{n}-S_{d}\right|+f_{a} \times\left|A_{n}-A_{d}\right|+f_{d} \times\left|\theta_{n}-\theta_{d}\right|+f_{q} \times Q+f_{n} \times N
$$

Where:

$f_{s}$
$f_{a}$
$f_{d}$
$f_{q}$
$f_{n}$
$S_{n}, A_{n}, \theta_{n}$
$S_{d}, A_{d}, \theta_{d}$
$Q$
$N$

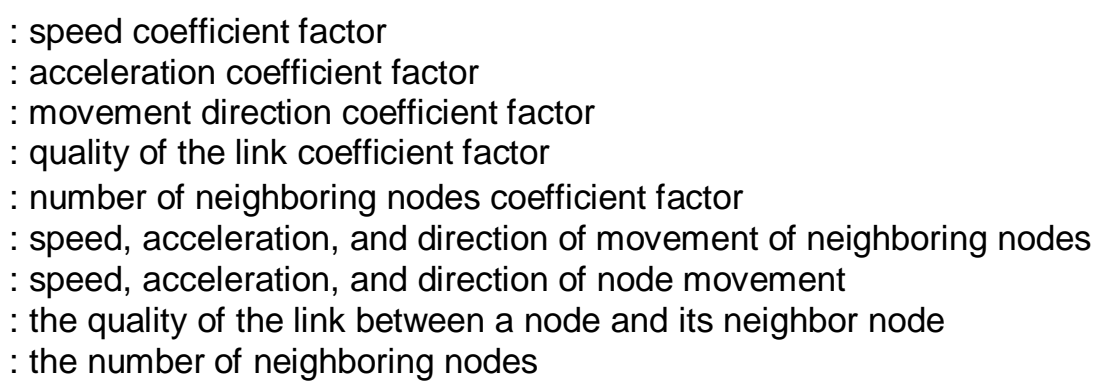

Total Weight of Route (TWR) is a formula used by Shen et al. and Arifiyan, Djanali and Anggoro in their research on PNT methods to find the best next-hop node to send packets. In this research TWR is used as a measure of the network condition of each node to calculate the value of the stability link which is used as a threshold for updating the radius value. The coefficients of each of the TWR factors used in this research are shown in Table 1.

Table 1. TWR Factors Coefficient

\begin{tabular}{ccc}
\hline No & Factor & Coefficient \\
\hline 1 & Speed & 50 \\
2 & Acceleration & 10 \\
3 & Movement Direction & 5 \\
4 & Quality of Link & 5 \\
5 & Number of Neighboring Node & 30 \\
\hline
\end{tabular}

(c) 2020 The Authors. Published by Universitas Muhammadiyah Malang

This is an open access article under the CC BY SA license. (https://creativecommons.org/licenses/by-sa/4.0/) 
Values calculated based on Equation 1 are stored in the routing table for each node as a stability link value. Each node will share their stability link value to other node using hello message. The average of stability link value from every neighbor node stored in routing table as a threshold value. When the radius update time arrives, the stability link along with the threshold value will be used as a reference to determine changes in the radius value, so that the radius value can increase or decrease to the limit of the radius value specified in the previous configuration. The changeable radius value refers to this network condition which creates dynamic zoning in ZRP so that the radius value of each node can change by adjusting to the network conditions as shown in Figure 1.

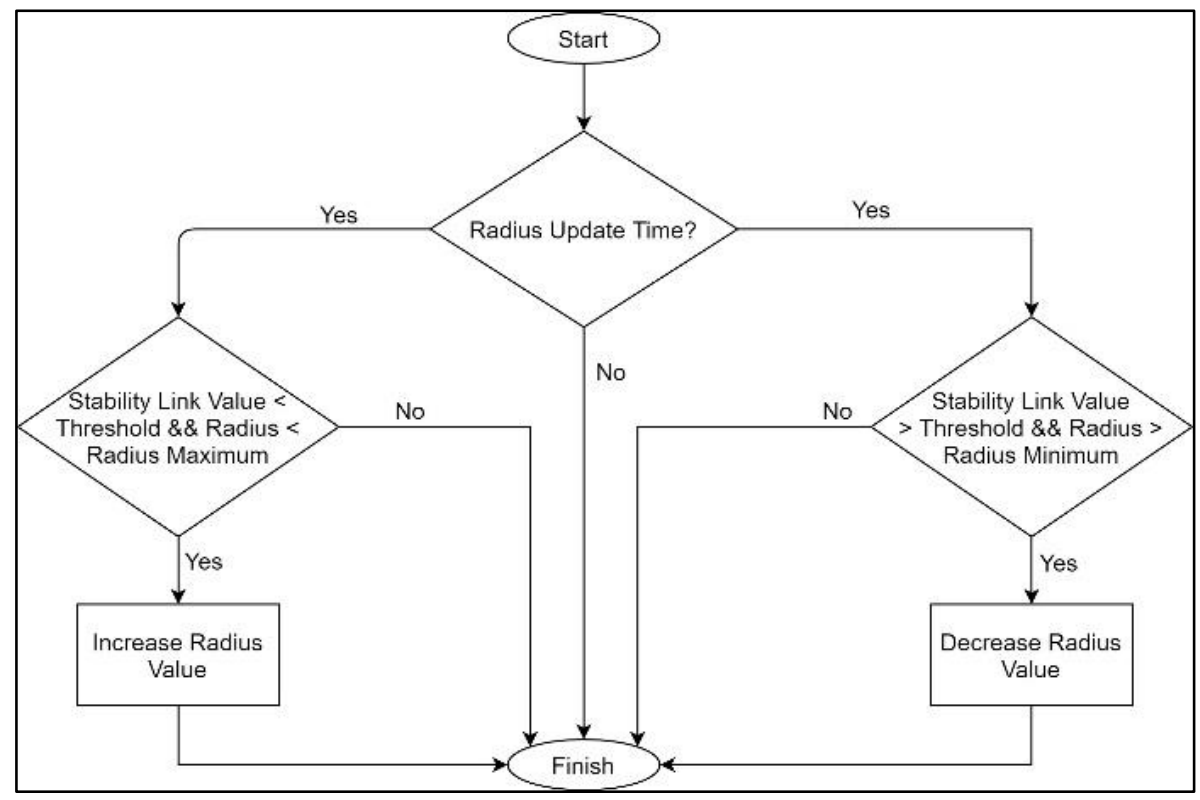

Figure 1. The Update Radius Value Process

Configuring the radius update time is important in terms of zoning dynamics in ZRP. Optimization is done so that the time to update the radius is not too fast or too slow. The radius update time that is too fast can make each node send too many packets in a short span of time so as to enable packet flooding. Conversely, if the radius update time is too slow, then the factor representation of the mobility of each node becomes less sensitive.

Simulations and tests are carried out to find the optimal radius update time. Tests carried out using NS-2 tools to conduct simulations with parameters as shown in the Table 2. The simulation on NS-2 is done by using variations in the number of nodes as a representation of the level of node density. The Simulation of Urban Mobility (SUMO) tool is used to create grid-based and real maps for simulations. Mobility of each node is also random based on the map that has been created with the help of the netgenerate feature of SUMO.

Table 2. Test and Simulations Parameter

\begin{tabular}{ccc}
\hline No & Parameter & Details \\
\hline 1 & Network Simulator & NS-2 version 2-35 \\
2 & Routing Protocol & Traditional ZRP, Zone Dynamics ZRP \\
3 & Radius Limit & Minimum 1 hop, Maximum 7 hops \\
4 & Numbers of Vehicle & $25,50,100,200$ vehicles \\
5 & Simulations Area & $1500 \mathrm{~m} \times 1500 \mathrm{~m}$ \\
6 & Radius of Traditional ZRP & 2 hops \\
7 & Radius Update Time & $5,10,15,20,25$ second \\
\hline
\end{tabular}

Analysis of the test results is carried out referring to the parameters of packet delivery ratio, end-to-end delay, and also routing overhead [26]. The proper radius update time can make ZRP have a high packet delivery ratio and reduce end-to-end delay and routing overhead that occur.

\section{Results and Discussion}

Tests and simulations are carried out in 2 stages, first is searching for the optimal update radius time and comparison of performance between traditional ZRP and zone dynamics ZRP. All tests are carried out with simulations in a grid-based and real map topology. 


\subsection{Proper Radius Update Time}

The first step is finding the optimal radius in the zone dynamics ZRP. The search was carried out by testing with the radius update time varias as mentioned in Table 2 from the test results, as shown in Table 3 and Figure 2, it can be seen that the radius update time affects the performance of the zone dynamics ZRP seen from the results of the packet delivery ratio (PDR). 25 seconds becomes the radius update time that produces the best PDR.
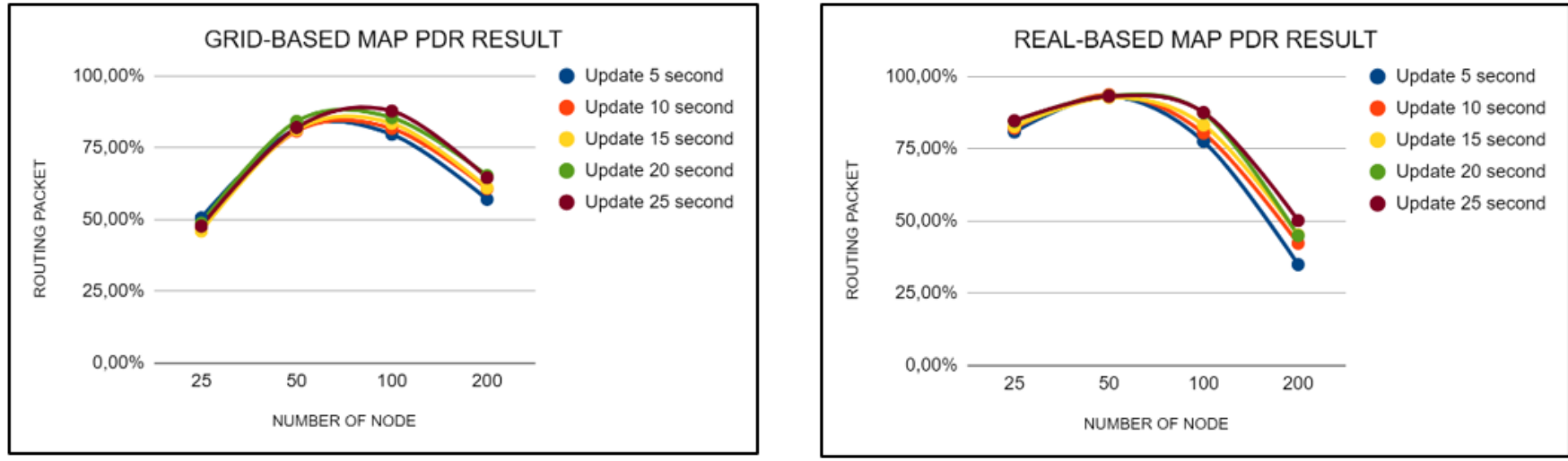

Figure 2. Packet Delivery Ratio Result

Table 3. Packet Delivery Ratio (PDR) Simulation Result

\begin{tabular}{ccccccccccc}
\hline \multirow{2}{*}{ Number of Vehicles } & \multicolumn{4}{c}{ Grid-Based Map Simulation } & \multicolumn{4}{c}{ Real-Based Map Simulation } \\
\cline { 2 - 11 } & $5 \mathrm{~s}$ & $10 \mathrm{~s}$ & $15 \mathrm{~s}$ & $20 \mathrm{~s}$ & $25 \mathrm{~s}$ & $5 \mathrm{~s}$ & $10 \mathrm{~s}$ & $15 \mathrm{~s}$ & $20 \mathrm{~s}$ & $25 \mathrm{~s}$ \\
\hline 25 & 50,52 & 47,05 & 45,89 & 48,65 & 47,61 & 80,75 & 82,07 & 82,64 & 84,50 & 84,68 \\
50 & 81,67 & 80,79 & 81,25 & 84,20 & 82,01 & 93,17 & 93,69 & 92,72 & 93,32 & 93,14 \\
100 & 79,63 & 81,65 & 83,49 & 85,47 & 87,79 & 77,48 & 80,33 & 83,19 & 87,34 & 87,54 \\
200 & 56,96 & 60,66 & 61,00 & 65,25 & 64,49 & 34,83 & 42,18 & 45,08 & 44,85 & 50,07 \\
\hline
\end{tabular}

The lower the end-to-end delay value, the better. The test results show that for end-to-end delay the 5 second radius update time shows the best results as shown in Table 4 and Figure 3 . While the 25 second radius update time that produces the best PDR actually produces the lowest end-to-end delay. In this test, end-to-end delay is calculated in millisecond (ms) units.
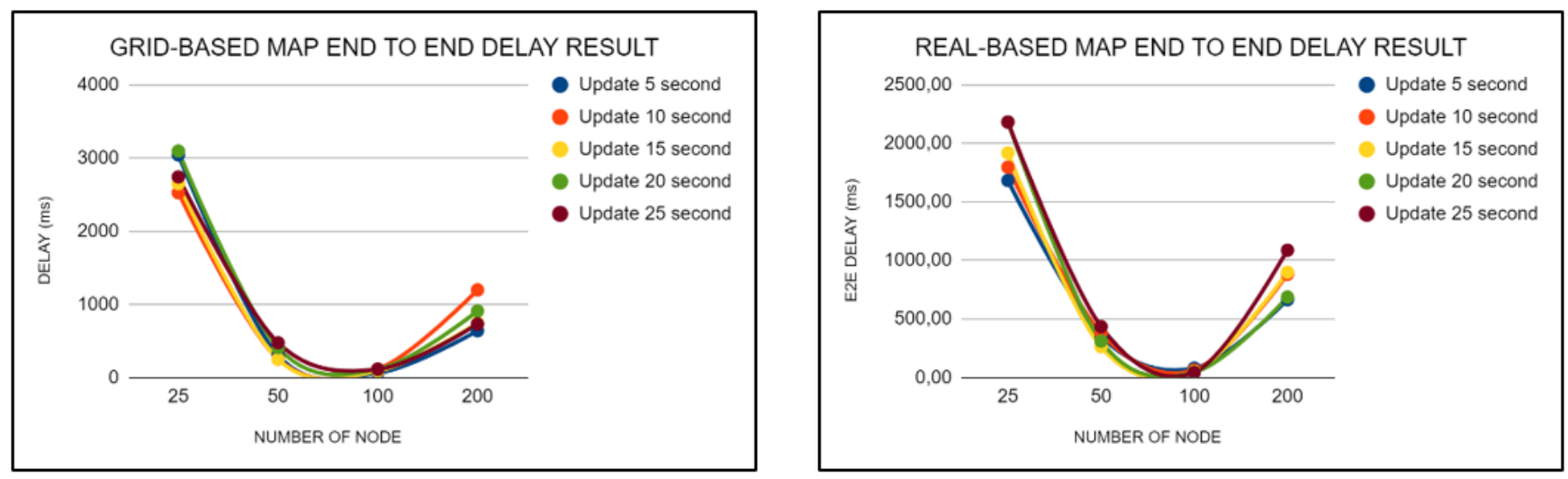

Figure 3. End-to-end Delay Result

Table 4. End-to-end Delay Simulation Result

\begin{tabular}{ccccccccccc}
\hline $\begin{array}{c}\text { Number } \\
\text { of }\end{array}$ & \multicolumn{4}{c}{ Grid-Based Map Simulation } & \multicolumn{5}{c}{ Real-Based Map Simulation } \\
\cline { 2 - 11 } Vehicles & $5 \mathrm{~s}$ & $10 \mathrm{~s}$ & $15 \mathrm{~s}$ & $20 \mathrm{~s}$ & $25 \mathrm{~s}$ & $5 \mathrm{~s}$ & $10 \mathrm{~s}$ & $15 \mathrm{~s}$ & $20 \mathrm{~s}$ & $25 \mathrm{~s}$ \\
\hline 25 & 3042,52 & 2525,40 & 2648,76 & 3094,70 & 2741,68 & 1683,08 & 1796,50 & 1918,22 & 2182,56 & 2182,05 \\
50 & 300,66 & 253,07 & 249,26 & 408,22 & 474,67 & 352,62 & 379,79 & 262,76 & 310,86 & 434,20 \\
100 & 61,59 & 115,75 & 87,54 & 112,76 & 112,21 & 79,69 & 64,00 & 50,59 & 44,49 & 43,49 \\
200 & 639,13 & 1196,64 & 744,15 & 908,03 & 730,20 & 664,25 & 881,36 & 898,51 & 686,38 & 1086,94 \\
\hline \hline
\end{tabular}

(c) 2020 The Authors. Published by Universitas Muhammadiyah Malang

This is an open access article under the CC BY SA license. (https://creativecommons.org/licenses/by-sa/4.0/) 
Kinetik: Game Technology, Information System, Computer Network, Computing, Electronics, and Control

The less the amount of routing overhead is better because it can reduce the burden on the network so that packet flooding does not occur. Table 5 and Figure 4 show a radius update time of 25 seconds producing the best routing overhead value in the simulations.
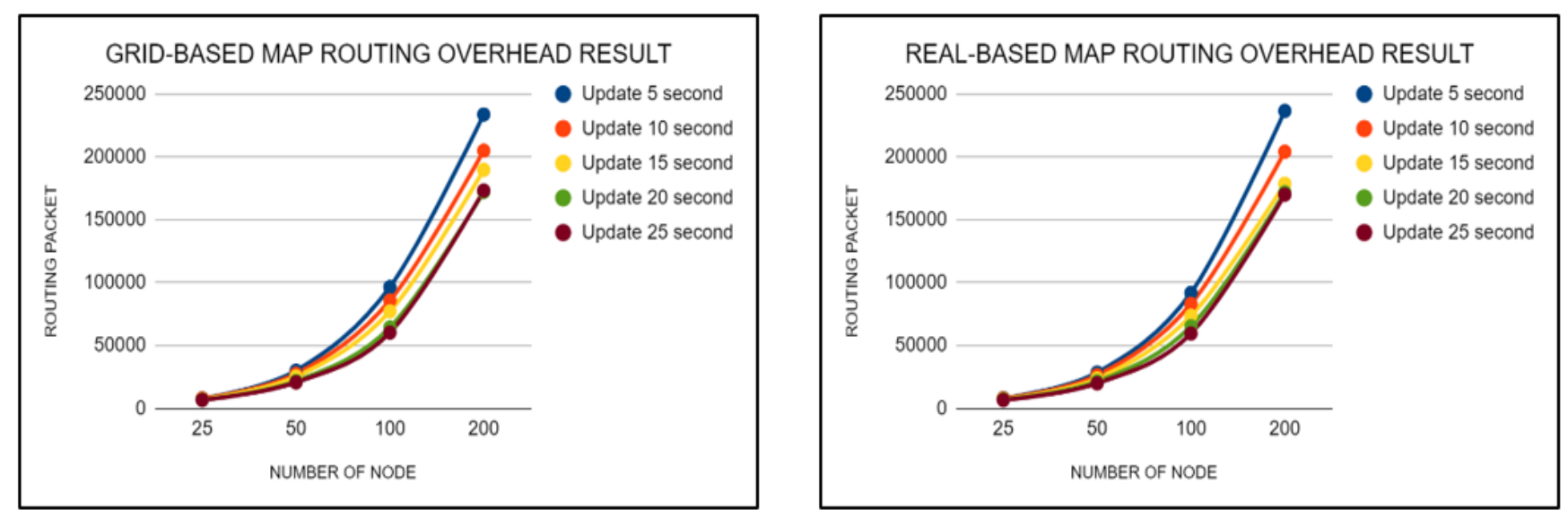

Figure 4. Routing Overhead Result

Table 5. Routing Overhead Simulation Result

\begin{tabular}{ccccccccccc}
\hline \multirow{2}{*}{$\begin{array}{c}\text { Number of } \\
\text { Vehicles }\end{array}$} & \multicolumn{4}{c}{ Grid-Based Map Simulation } & \multicolumn{4}{c}{ Real-Based Map Simulation } \\
\cline { 2 - 12 } & $5 \mathrm{~s}$ & $10 \mathrm{~s}$ & $15 \mathrm{~s}$ & $20 \mathrm{~s}$ & $25 \mathrm{~s}$ & $5 \mathrm{~s}$ & $10 \mathrm{~s}$ & $15 \mathrm{~s}$ & $20 \mathrm{~s}$ & $25 \mathrm{~s}$ \\
\hline 25 & 7968 & 7837 & 7227 & 6784 & 6678 & 8175 & 7884 & 7377 & 7004 & 6605 \\
50 & 29924 & 27471 & 25228 & 21651 & 20639 & 28494 & 26314 & 23708 & 21381 & 19861 \\
100 & 96443 & 85829 & 77134 & 64357 & 60215 & 91863 & 83053 & 73589 & 65253 & 59486 \\
200 & 233726 & 204905 & 189715 & 172227 & 173236 & 236561 & 204118 & 178628 & 171746 & 170002 \\
\hline
\end{tabular}

It can be concluded that the timing of the radius update is very influential on ZRP performance. The 25 second radius update time produces the best PDR value and routing overhead, but it produces the lowest delay. The 5 second radius update time produces the best end-to-end delay.

\subsection{Comparation Between Traditional ZRP and Zone Dynamics ZRP}

In the second stage of the test, the zone dynamics ZRP performance with the optimized radius update time compared to the traditional ZRP performance. A radius update time of 25 seconds is used because it is rated as the most optimal time for the best performance of zone dynamics ZRP.

In terms of PDR, zone dynamics ZRP performance is quite equal when compared to traditional ZRP. As can be seen in Figure 5 and Table 6 , at low density ( 25 \& 50 vehicles) and medium density ( 100 vehicles) the traditional ZRP produces better PDR, but at high density environment the zone dynamics ZRP with radius update time that have been optimized has better PDR.
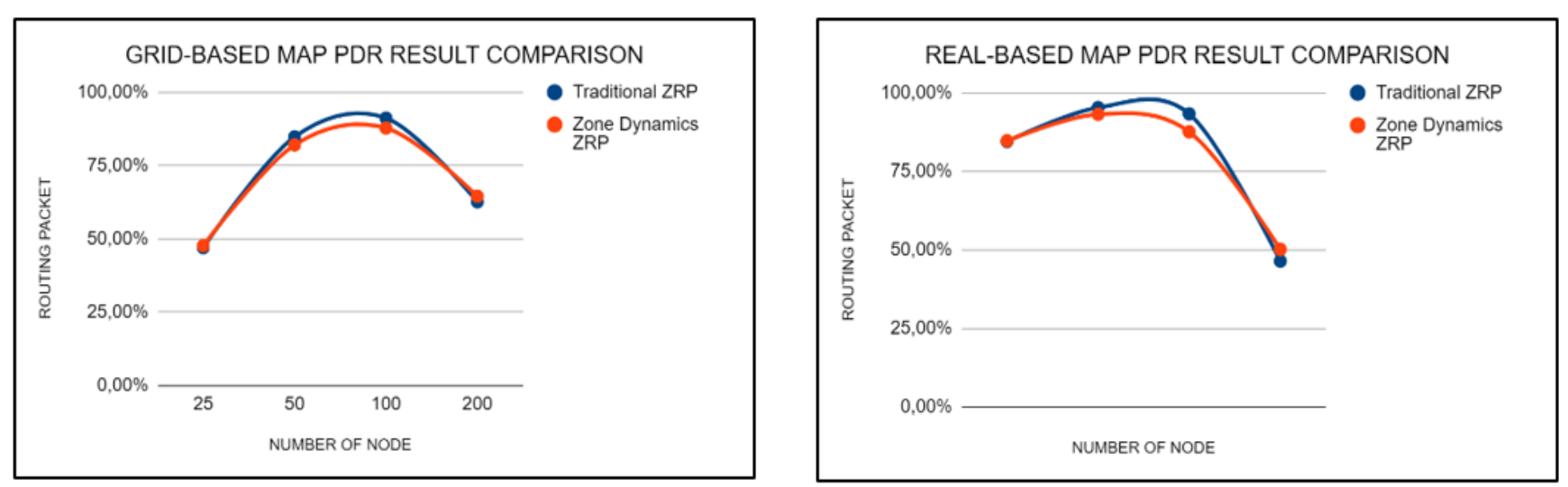

Figure 5. PDR Comparisons Result 
Kinetik: Game Technology, Information System, Computer Network, Computing, Electronics, and Control Table 6. PDR Comparisons of Traditional ZRP and Zone Dynamics ZRP

\begin{tabular}{ccccc}
\hline \multirow{2}{*}{ Number of Vehicles } & \multicolumn{2}{c}{ Grid-Based Map Simulation } & \multicolumn{2}{c}{ Real-Based Map Simulation } \\
\cline { 2 - 5 } & Traditional ZRP & Zone Dynamics ZRP & Traditional ZRP & Zone Dynamics ZRP \\
\hline 25 & 55,41 & 47,61 & 84,47 & 84,68 \\
50 & 84,80 & 82,01 & 95,26 & 93,14 \\
100 & 91,19 & 87,79 & 93,27 & 87,54 \\
200 & 62,60 & 64,49 & 46,34 & 50,07 \\
\hline
\end{tabular}

Figure 6 and Table 7 show the comparison results of traditional ZRP and zone dynamics ZRP performance in terms of end-to-end delay. It can be seen that zone dynamics ZRP performs better than traditional ZRP. A smaller delay is generated by zone dynamics ZRP at all density levels. The only exception results occur at low density levels in simulation with real-based maps when the traditional ZRP has lower delay.
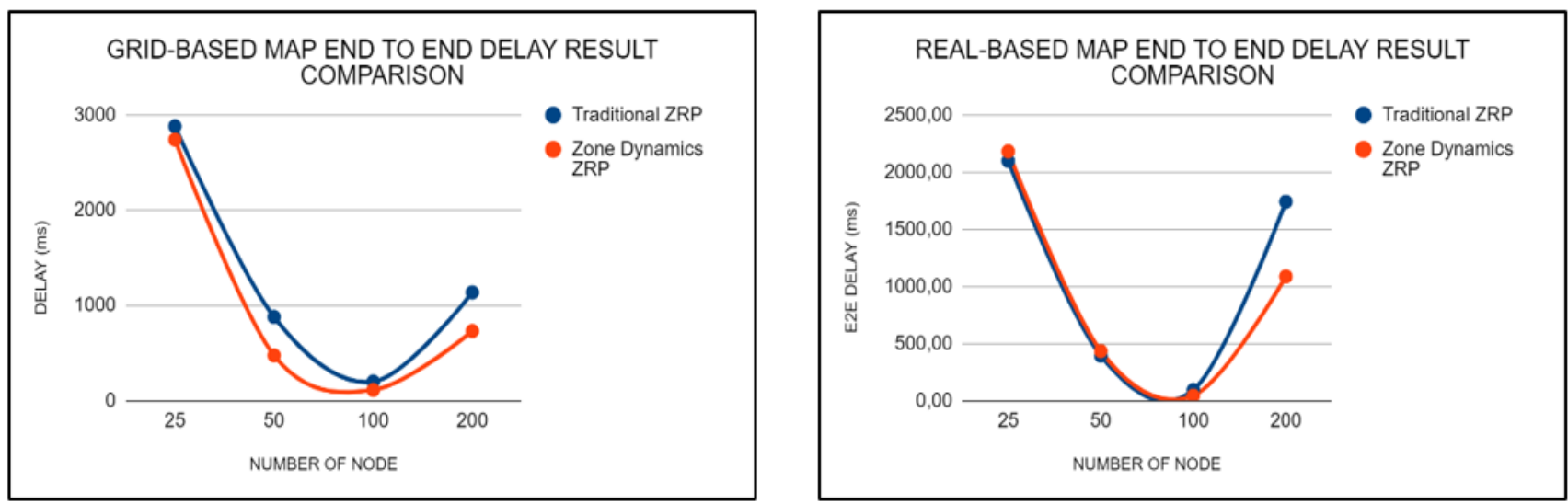

Figure 6. End-to-end Delay Comparisons Result

Table 7. End-to-end Delay Comparisons of Traditional ZRP and Zone Dynamics ZRP

\begin{tabular}{ccccc}
\hline \multirow{2}{*}{ Number of Vehicles } & \multicolumn{2}{c}{ Grid-Based Map Simulation } & \multicolumn{2}{c}{ Real-Based Map Simulation } \\
\cline { 2 - 5 } & Traditional ZRP & Zone Dynamics ZRP & Traditional ZRP & Zone Dynamics ZRP \\
\hline 25 & 2881,62 & 2741,68 & 2098,88 & 2182,05 \\
50 & 878,22 & 474,67 & 393,77 & 434,20 \\
100 & 198,91 & 112,21 & 92,72 & 43,49 \\
200 & 1136,72 & 730,20 & 1740,66 & 1086,94 \\
\hline
\end{tabular}

Figure 7 and Table 8 show the comparison of routing overhead generated by traditional ZRP and zone dynamics ZRP. The routing overhead from zone dynamics ZRP is better than traditional ZRP. The lesser amount of routing overhead will make the network not burdened by packets sent and reduce the possibility of packet flooding.
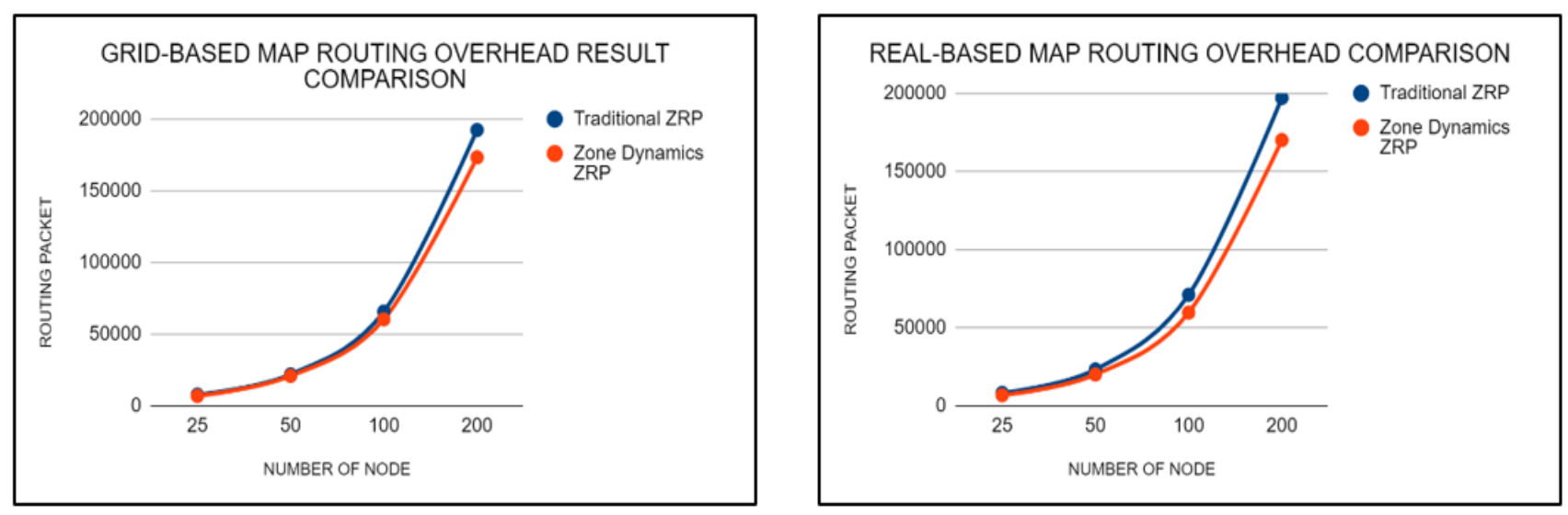

Figure 7. Routing Overhead Comparisons Result

(c) 2020 The Authors. Published by Universitas Muhammadiyah Malang

This is an open access article under the CC BY SA license. (https://creativecommons.org/licenses/by-sa/4.0/) 
Table 8. Routing Overhead Comparisons of Traditional ZRP and Zone Dynamics ZRP

\begin{tabular}{ccccc}
\hline \multirow{2}{*}{ Number of Vehicles } & \multicolumn{2}{c}{ Grid-Based Map Simulation } & \multicolumn{2}{c}{ Real-Based Map Simulation } \\
\cline { 2 - 5 } & Traditional ZRP & Zone Dynamics ZRP & Traditional ZRP & Zone Dynamics ZRP \\
\hline 25 & 7538 & 6678 & 8023 & 6605 \\
50 & 21676 & 20639 & 23058 & 19861 \\
100 & 65565 & 60215 & 70918 & 59486 \\
200 & 192356 & 173236 & 197029 & 170002 \\
\hline
\end{tabular}

The results of tests that have been done show that optimizing the time of the radius update can improve the performance of the zone dynamics ZRP. Comparisons made also showed that zone dynamics ZRP with optimal radius update time were able to work better than traditional ZRP.

\section{Conclusion}

Optimization at the time of radius update can improve the performance of ZRP dynamics zones. Simulation and test results show improved performance in zone dynamics ZRP which the radius update time has been optimized. Optimization of the radius update time makes the zone dynamics ZRP perform better than traditional ZRP. Optimization of other factors might also be done to make further performance improvement.

\section{References}

[1] A. Nasipuri, 'Mobile Ad Hoc Networks', Pp. 1-26, 2004. https://doi.org/10.1016/B978-075067695-3/50005-7

[2] L. Raja and S. Baboo, 'An Overview of MANET: Applications, attacks and challenges', Int. J. Comput. Sci. Mob. Comput., Vol. 3131, No. 1, Pp. 408-417, 2014.

[3] J. Hoebeke, I. Moerman, B. Dhoedt, and P. Demeester, 'An overview of mobile ad hoc networks: Applic ations and challenges', Commun. Netw., Vol. 2, No. 1, Pp. 87-110, 2010

[4] F. Li and Y. Wang, 'Routing in Vehicular Ad Hoc Networks: A Survey', IEEE VEHICULAR TECHNOLOGY MAGAZINE, No. June, Pp. 12-22, 2007. https://doi.org/10.1109/MVT.2007.912927

[5] S. Yousefi, M. S. Mousavi, and M. Fathy, 'Vehicular Ad hoc Networks (VANETs): Challenges and perspectives', in ITST 2006 - 2006 6th International Conference on ITS Telecommunications, Proceedings, Pp. 761-766, 2007. https://doi.org/10.1109/ITST.2006.289012

[6] V. N. Jayanti, 'Routing Protocols in MANET', Int. J. Comput. Sci. Mob. Comput., Vol. 3, No. 7, Pp. 119-125, 2014.

[7] N. Beijar, 'Zone Routing Protocol ( ZRP )', Pp. 1-12, 2002.

[8] Z. J. Haas and M. R. Pearlman, 'On the Performance of a Routing Protocol for the Reconfigurable Wireless Networks', J. Appl. Stat., Vol. 11, No. 2, Pp. 1-4, 1998. https://doi.org/10.1109/VETEC.1998.686483

[9] N. Jain and Y. Chaba, 'Simulation based Performance Analysis of Zone Routing Protocol in Manet', Int. J. Comput. Appl., Vol. 88, No. 4, Pp. 47-52, 2014. https://doi.org/10.5120/15344-3684

[10] T. C. Huang, W. C. Cheng, and L. Tang, 'An effective scheme for detecting articulation points in zone routing protocol', Lect. Notes Inst. Comput. Sci. Soc. Telecommun. Eng., Vol. 98 LNICST, Pp. 521-533, 2012. https://doi.org/10.1007/978-3-642-30493-4 50

[11] M. R. Pearlman and Z. J. Haas, 'Determining the optimal configuration for the zone routing protocol', IEEE J. Sel. Areas Commun., Vol. 17, No. 8, Pp. 1395-1414, 1999. https://doi.org/10.1109/49.779922

[12] T. Yélémou, B. Zerbo, M. T. Dandjinou, and O. Sie, 'Impact of ZRP Zone Radius Value on Wireless Network Performance Tiguiane', Vol. 92, Pp. 164-173, 2012. https://doi.org/10.1007/978-3-030-16042-5_16

[13] Chenn-Jung Huang, Liang-Chun Chen, Yao-Chuan Lin, Yi-Ta Chuang, Wei Kuang Lai, and Sheng-Yu Hsiao, 'A Zone Routing Protocol for Bluetooth MANET with Online Adaptive Zone Radius', in International Conference on Information Communications \& Signal Processing, Pp. 579-583, 2006. https://doi.org/10.1109/ICICS.2005.1689113

[14] P. Samar, M. R. Pearlman, and Z. J. Haas, 'Independent Zone Routing: An Adaptive Hybrid Routing Framework for Ad Hoc Wireless Networks', IEEE/ACM Trans. Netw., Vol. 12, No. 4, Pp. 529-560, 2004. https://doi.org/10.1109/TNET.2004.833153

[15] X. Yang, Q. Chen, C. Chen, and J. Zhao, 'Improved ZRP Routing Protocol Based on Clustering', Procedia Comput. Sci., Vol. 131, Pp. 9921000, 2018. https://doi.org/10.1016/j.procs.2018.04.242

[16] Y. Oigawa and F. Sato, 'Improvement in IntErzone Routing Protocol of ZRP Based on Bloom Filter', J. Inf. Process., Vol. 26, No. 0, Pp. 124131, 2018. https://doi.org/10.2197/ipsjjip.26.124

[17] J. Zhou, Y. Cheng, and J. Lu, 'Velocity Based Adaptive Zone Routing Protocol', in International Symposium on Intelligent Signal Processing and Communication Systems, Pp. 337-340, 2007. https://doi.org/10.1109/ISPACS.2007.4445892

[18] A. K. Jaiswal and P. Singh, 'Optimizing velocity based adaptive zone routing protocol', in 2010 International Conference on Computer and Communication Technology, ICCCT-2010, Pp. 149-152, 2010. https://doi.org/10.1109/ICCCT.2010.5640413

[19] K. Lakhtaria, 'Analyzing Zone Routing Protocol in MANET Applying Authentic Parameter Analyzing Zone Routing Protocol in MANET Applying Authentic Parameter', No. May, 2014.

[20] S. Sinha and B. Sen, 'Effect of Varying Node Density and Routing Zone Radius in ZRP : A Simulation Based Approach', Int. J. Comput. Sci. Eng., Vol. 4, No. 06, Pp. 1069-1077, 2012.

[21] X. Zheng, Q. Qi, Q. Wang, and Y. Li, 'An Adaptive Density-based Routing Protocol for Flying Ad Hoc Networks', Vol. 040113, No. October, 2017. https://doi.org/10.1063/1.5005315

[22] E. Natsheh and K. Buragga, 'Density Based Routing Algorithm for Spare / Dense Topologies in Wireless Mobile Ad-Hoc Networks', Vol. 3, No. 2, Pp. 312-319, 2010.

[23] A. Wang, H. Chao, and S. Member, 'Direction Density-Based Secure Routing Protocol for Healthcare Data in Incompletely Predictable Networks', IEEE Access, Vol. 4, Pp. 9163-9173, 2017. https://doi.org/10.1109/ACCESS.2016.2637887

[24] X. Shen, Y. Wu, Z. Xu, and X. Lin, 'AODV-PNT : An Improved Version of AODV Routing Protocol with Predicting Node Trend in VANET', in The 7th IEEE/International Conference on Advanced Infocomm Technology, Pp. 91-97, 2014. https://doi.org/10.1109/ICAIT.2014.7019536

Cite: Fadhillah, M., \& Anggoro, R. (2020). Time Optimization for Radius Updates in Zone Dynamics of Zone Routing Protocol. Kinetik: Game Technology, Information System, Computer Network, Computing, Electronics, and Control, 5(2). doi:https://doi.org/10.22219/kinetik.v5i2.1064 
[25] G. Arifiyan, S. Djanali, and R. Anggoro, 'AODV-PNT Performance Study with Added Factor Number of Neighbor Nodes on VANET', in The 3rd International Seminar on Science and Technology, Pp. 69-71, 2017. http://dx.doi.org/10.12962/j23546026.y2018i1.3510

[26] R. Astya, P. Nand, and S. C. Sharma, 'Parametric analysis of zone routing protocol', Commun. Comput. Inf. Sci., Vol. 169 CCIS, No. 91, Pp. 247-251, 2011. https://doi.org/10.1007/978-3-642-22577-2_33 